\title{
Abnormal quality detection and isolation in water distribution networks using simulation models
}

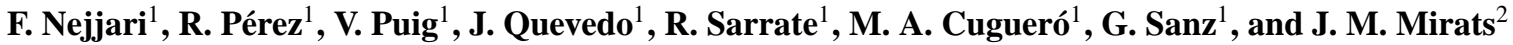 \\ ${ }^{1}$ Technical University of Catalonia (UPC), 10 Rambla Sant Nebridi, 08222 Terrassa, Spain \\ ${ }^{2}$ CETaqua Water Technology Centre, Crta. d'Esplugues n.75, 08940 Cornellà de Llobregat, Spain
}

Correspondence to: F. Nejjari (fatiha.nejjari@upc.edu)

Received: 29 June 2012 - Published in Drink. Water Eng. Sci. Discuss.: 10 August 2012

Revised: 11 October 2012 - Accepted: 16 October 2012 - Published: 16 November 2012

\begin{abstract}
This paper proposes a model based detection and localisation method to deal with abnormal quality levels based on the chlorine measurements and chlorine sensitivity analysis in a water distribution network. A fault isolation algorithm which correlates on line the residuals (generated by comparing the available chlorine measurements with their estimations using a model) with the fault sensitivity matrix is used. The proposed methodology has been applied to a District Metered Area (DMA) in the Barcelona network.
\end{abstract}

\section{Introduction}

Drinking water distribution networks are complex large-scale systems that are designed to supply clean water to consumers at any time based on demand. To guarantee the high quality of water, chlorine is certainly the most common disinfectant used in these systems. The maintenance of chlorine residual is needed at all the points in the network. Its propagation and level are affected by both bulk and pipe wall reactions. Water quality fault will result in reduction of residual oxidant and can be manifested in added variability and lower chlorine concentrations at sensor locations in the network. This variability of chlorine concentration in the network would allow differentiating normal from anomalous water quality conditions.

The water distribution network can be perceived as a complex chemical reactor in which various processes occur simultaneously (Sadiq et al., 2003). Some of these processes take place in the bulk phase and others on the pipe's wall, and all of them can degrade water quality. Water quality faults can be caused directly or indirectly by an internal corrosion due to an oxidation/reduction reaction, by detachment and leaching of pipe material or biofilm formation (LeChevallier et al., 1987), by regrowth of microorganisms on the internal surface and/or by a loss of disinfectant and formation of byproducts (DBPs), or an intrusion of contaminants (Lindley, 2001).
This paper proposes a detection and localisation method for abnormal water quality levels based on chlorine measurements and chlorine sensitivity analysis of the nodes in a network. Simulations of the water quality of the network performed both for realistic and abnormal bulk decay at a certain time in each pipe segment (link) provides an approximation of this sensitivity. A fault isolation algorithm that correlates the residuals (generated by comparing the available chlorine concentration measurements with their estimated values using a model) with the fault sensitivity matrix is used. The correlation between the observed residual fault signature and each column of the sensitivity matrix is a measure of the similarity of the residual effect concerning pipe bulk decay fault due to pipe material detachment. The proposed approach is illustrated by a simulation example, based on a real water distribution network. Water quality data were obtained by simulation using EPANET 2.0 software, which can track the chlorine decay evolution through the water distribution system. The same methodology has been applied to detect and isolate leakages in nodes of the same network (Quevedo et al., 2011). In addition, a comparative study of this technique based on correlation analysis and the one based on a binary matrix has been done in Pérez et al. (2011). Correlation analysis is also an important tool for data validation. Further good results, using the same methodology to determine flow demand patterns given some metered data from 
Barcelona water distribution network, are shown in Quevedo et al. (2010). Nevertheless, the application to abnormal water quality detection introduces irrelevant dynamics in leakage detection achieved using quasi-static models.

This paper is organised as follows: in Sect. 2, the methodology used for water quality diagnosis is presented. In Sect. 3, the proposed approach is demonstrated by its application on a DMA of the Barcelona water distribution network. Finally, in Sect. 4 some conclusions are given.

\section{Water quality fault diagnosis}

\subsection{Fault diagnosis procedure}

Model based fault diagnosis consists in the process of detecting and isolating a fault by using analytical redundancy techniques to monitor the changes in the dynamics of a system (Gertler, 1998). The consistency check is based on residuals $r(k)$ computation, obtained from measured input $u(k)$ and output $y(k)$ signals and the analytical relationships which are obtained by system modelling that provide an estimated output $\hat{y}(k)$ :

$r(k)=y(k)-\hat{y}(k)$

At each time step $k$, the residual is compared with a threshold value (zero in the ideal case and almost zero in a real case). The threshold value is typically determined using statistical or set-based methods that take into account the effect of noise and model uncertainty (Blanke et al., 2006). When a residual is bigger than the threshold, it is determined that there is a fault in the system; otherwise, it is considered that the system is working properly. In practice, because of input/output noise, nuisance inputs and modelling errors affecting the model considered, robust residual generators must be used. Robustness can be achieved at the residual generation phase (active) or at the evaluation phase (passive). Robust residual evaluation allows obtaining a set of observed fault signatures

$\Phi(k)=\left[\phi_{1}(k), \phi_{2}(k), \ldots \phi_{n \phi}(k)\right]$

where each fault indicator is obtained as follows:

$\phi_{i}(k)=\left\{\begin{array}{l}0 \text { if }\left|r_{i}(k)\right| \leq \tau_{i}(k) \\ 1 \text { if }\left|r_{i}(k)\right|>\tau_{i}(k)\end{array}\right.$

and where $\tau_{i}(k)$ is the threshold associated to the residual $r_{i}(k)$.

\subsection{Fault localisation using the correlation method}

Fault isolation is carried out on the basis of observed fault signatures, $\phi_{i}(k)$, generated by the detection module and its relation with all the considered faults, $f(k)=$ $\left[f_{1}(k), f_{2}(k), \ldots f_{n f}(k)\right]$, which are compared with the theoretical fault signature matrix.
The use of the information associated with the relationship between the residuals and faults, by means of the residual fault sensitivity, allows improving the isolation results. Sensitivity Matrix S (Eq. 3) provides the sensitivity of the chlorine sensor residuals for each different fault $f_{j}$ affecting the system:

$$
\mathbf{S}=\begin{gathered}
r_{1} \\
r_{2} \\
\vdots \\
r_{n}
\end{gathered}\left[\begin{array}{cccc}
f_{1} & f_{2} & \cdots & f_{m} \\
s_{11} & s_{12} & \cdots & s_{1 m} \\
s_{21} & s_{22} & \cdots & s_{2 m} \\
\vdots & \vdots & \ddots & \vdots \\
s_{n 1} & s_{n 2} & \cdots & s_{n m}
\end{array}\right]
$$

where $s_{i j}=\frac{\mathrm{C}_{1 f j}-C_{1 n f}}{f_{j}}$ with $\mathrm{C}_{1 f j}$ and $C_{1 n f}$ being the mean values of the chlorine concentration $C_{1}$ in node $j$ at a certain time, when the bulk decay is abnormal or normal, respectively.

The main idea of correlation-based fault diagnosis consists in comparing the columns (faults) of the sensitivity matrix $\mathbf{S}$ (Eq. 3) with the corresponding residual vector at time $k$ by using the correlation function. The correlation coefficient $\rho_{r, S_{f j}}(k)$ between $r(k)$ and each column $j$ of $S$ (i.e. $S_{f j}(k)$ ) is computed as the Pearson's correlation coefficient, that is defined as:

$\rho_{r, S_{f j}}=\frac{\operatorname{cov}\left(r, S_{f j}\right)}{\sqrt{\operatorname{cov}(r, r) \operatorname{cov}\left(S_{f j}, S_{f j}\right)}}$

where $\operatorname{cov}\left(r, S_{f j}\right)=E\left[(r-\bar{r})\left(S_{f j}-\bar{S}_{f j}\right)\right]$ is the covariance function between $r$ and $S_{f j}$ being $\bar{r}=E(r)$ and $\bar{S}_{f j}=E\left(S_{f j}\right)$, respectively.

The columns of $\mathbf{S}$ having higher correlation values with the residual vector at time $k$ are the most probable elements to have a fault. The correlation between the observed residual fault signature (i.e. $r(k)$ ) and each column of the matrix $S$ is a measure of the similarity between the real fault residual effect (with unknown magnitude) and the faults considered in matrix $\mathbf{S}$ (Eq. 3) (with known magnitude) that allows discovering which is the column of this matrix (fault) having the same behaviour. For faults with similar size to those used to obtain the sensitivity matrix $\mathbf{S}$, the correlation function obtains the maximum similarity (shape and form), i.e. $\rho=1$ in the element having the fault, for any magnitude of the real fault. Because of the non-linearity of the water network system, if the real magnitude of the fault is far from the fault size used to compute matrix $\mathbf{S}$, the similarity of the correlation function decreases but a high correlation between the residuals corresponding to a particular fault and the corresponding column of the sensitivity matrix still exists.

The vector obtained is the decision vector that will be used to figure out which is the fault occurring in the system. More concretely, the maximum correlation value in this vector will point out the corresponding $\mathbf{S}$ column (fault) as the most probable element to be faulty:

$\max _{j}\left(\rho_{r, S_{f_{j}}}(k)\right)$ 


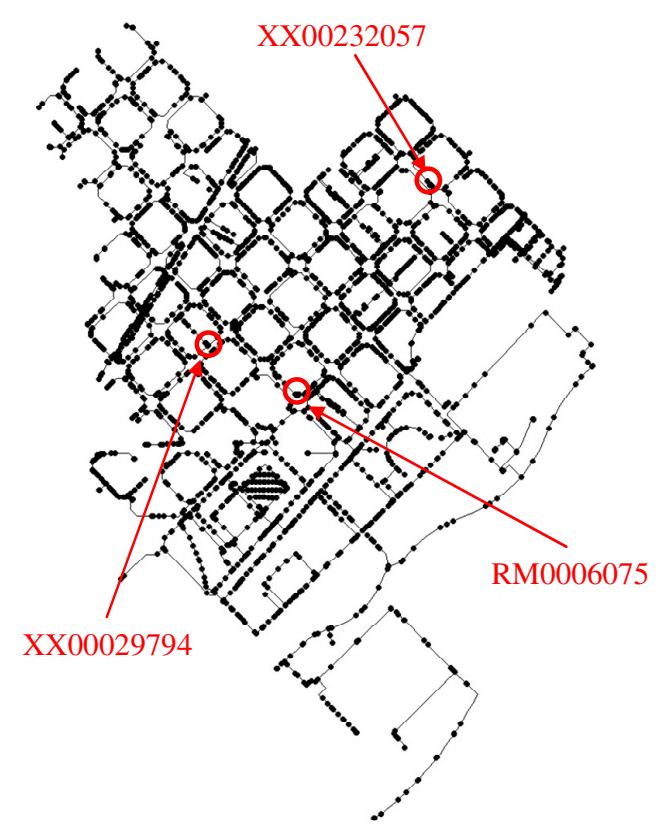

Figure 1. The layout of Nova Icària Network with the 3 installed chlorine sensor.

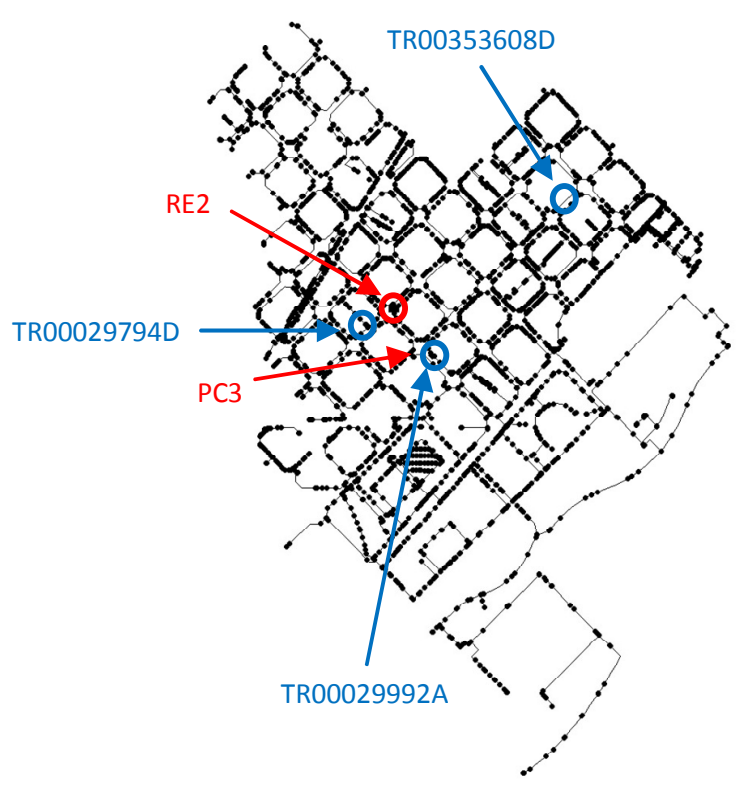

Figure 2. Location of chlorine injectors (red) and links with chlorine decay fault (blue).

\section{Application}

A DMA of a real water distribution network (Fig. 1) was used to verify the proposed method for abnormal water quality detection and location. This DMA, located in Barcelona's Nova Icària area, is included in the 55th pressure level within the city network and has 1996 nodes and 3442 pipes. The DMA

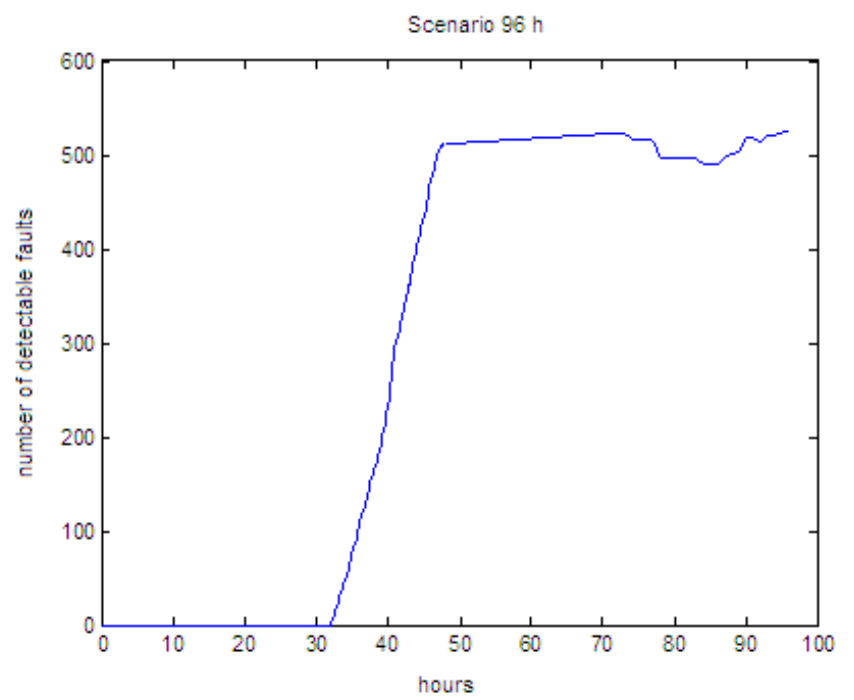

Figure 3. Number of bulk chlorine decay faults using 3 preinstalled sensors.

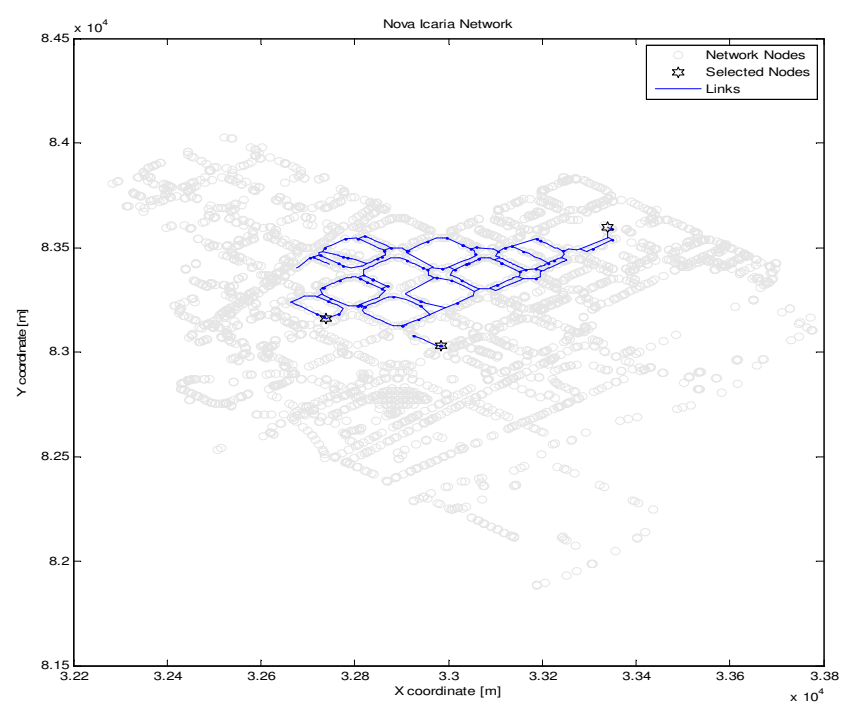

Figure 4. Geographic distribution of faults using 3 pre-installed sensors.

has two inputs, called Alaba and Llull, and three chlorine sensors, which are also shown in Fig. 1.

Scenarios have been generated using EPANET simulation software, considering these three sensors already installed in the network (see Fig. 1). The events have been generated setting the Initial Quality and Source Quality parameters of the injectors Alaba (RE) and Llull (PC3) in Fig. 2. The scenarios have been generated changing the $K_{\text {bulk }}$ parameter at the corresponding links of the network at 08:00 a.m of day 2 (32nd hour of the episode). The values of faulty/non-faulty behaviours on these links are $K_{\text {bulk }}=-2.3064151 / d$ for the non-faulty mode and $K_{\text {bulk }}=-501 / d$ for the faulty mode. 

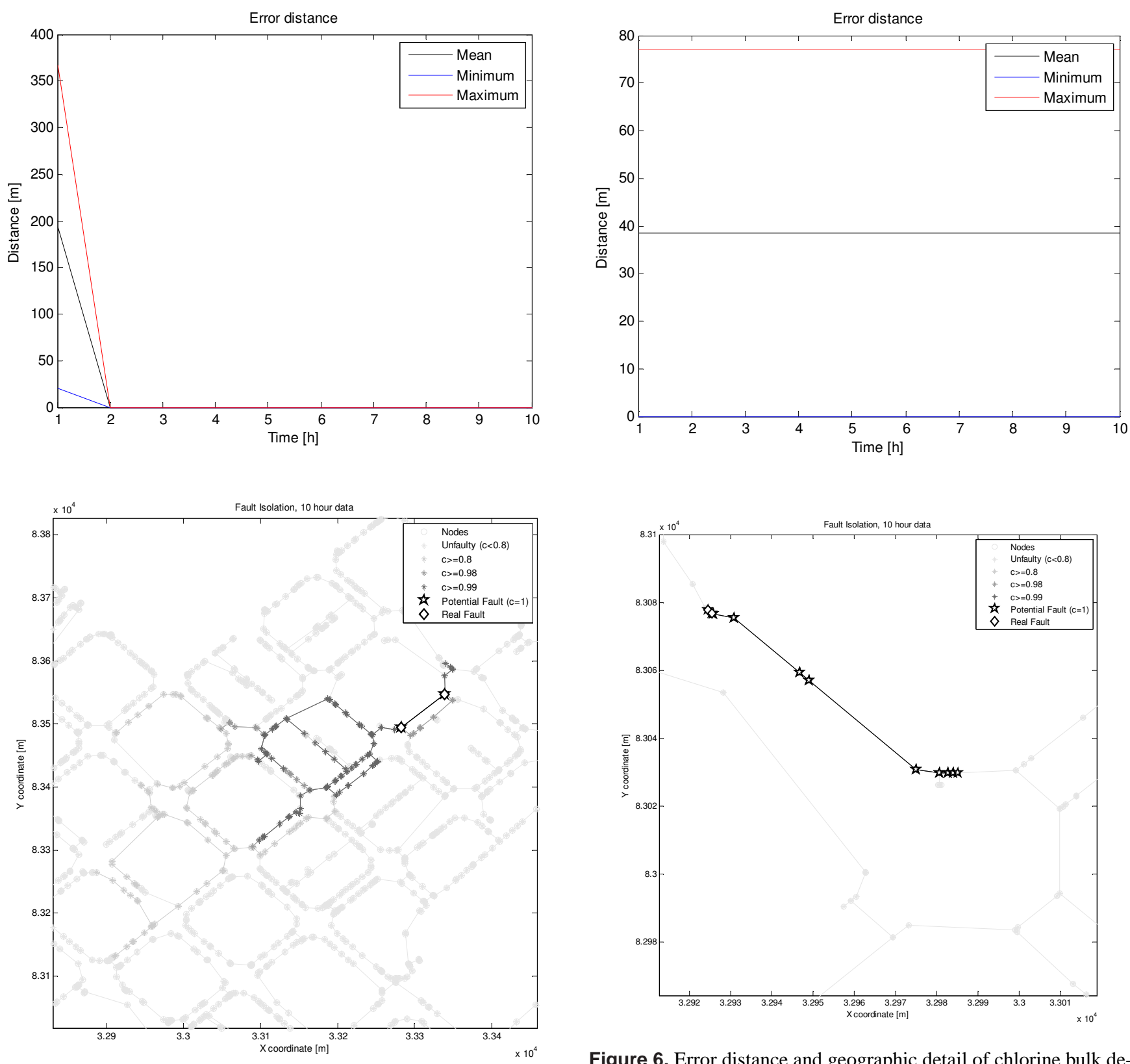

Figure 5. Error distance and geographic detail of chlorine bulk decay fault detection for the link TR00353608D at hour 48 .

The scenario has been run for $48 \mathrm{~h}$ and data sets have been created according to these scenarios.

Figure 3 shows the number of bulk chlorine decay faults that may be detected using sensors located in Fig. 1 after $96 \mathrm{~h}$ of simulation. It may be seen how, with the sensors already installed, the number of faults that may be detected is about 500. These links are distributed geographically as shown in Fig. 4.

Three different links of the network, included in the set of detectable faults in Fig. 3, are considered to generate the faulty scenarios. These links labelled "TR00353608D",

Figure 6. Error distance and geographic detail of chlorine bulk decay fault detection for the link TR00029992A at hour 48 .

“TR00029992A" and “TR00029794D”, respectively are shown in Fig. 2. The simulated data are obtained using the methodology presented. Figures 8-10 show the fault detection results obtained at hour 48 for every chlorine bulk decay fault. In these figures, the link with the actual fault is represented with a diamond, and the starred nodes are the potential faulty nodes suggested by the correlation method. The rest of the nodes are divided in grey-scaled areas, depending on the correlation value they have: the most correlated with the fault signature, the darkest the area they are located. The evolution of the error distance through the period of results generation (15 to $24 \mathrm{~h}$ ) and a detail of the detection for each 

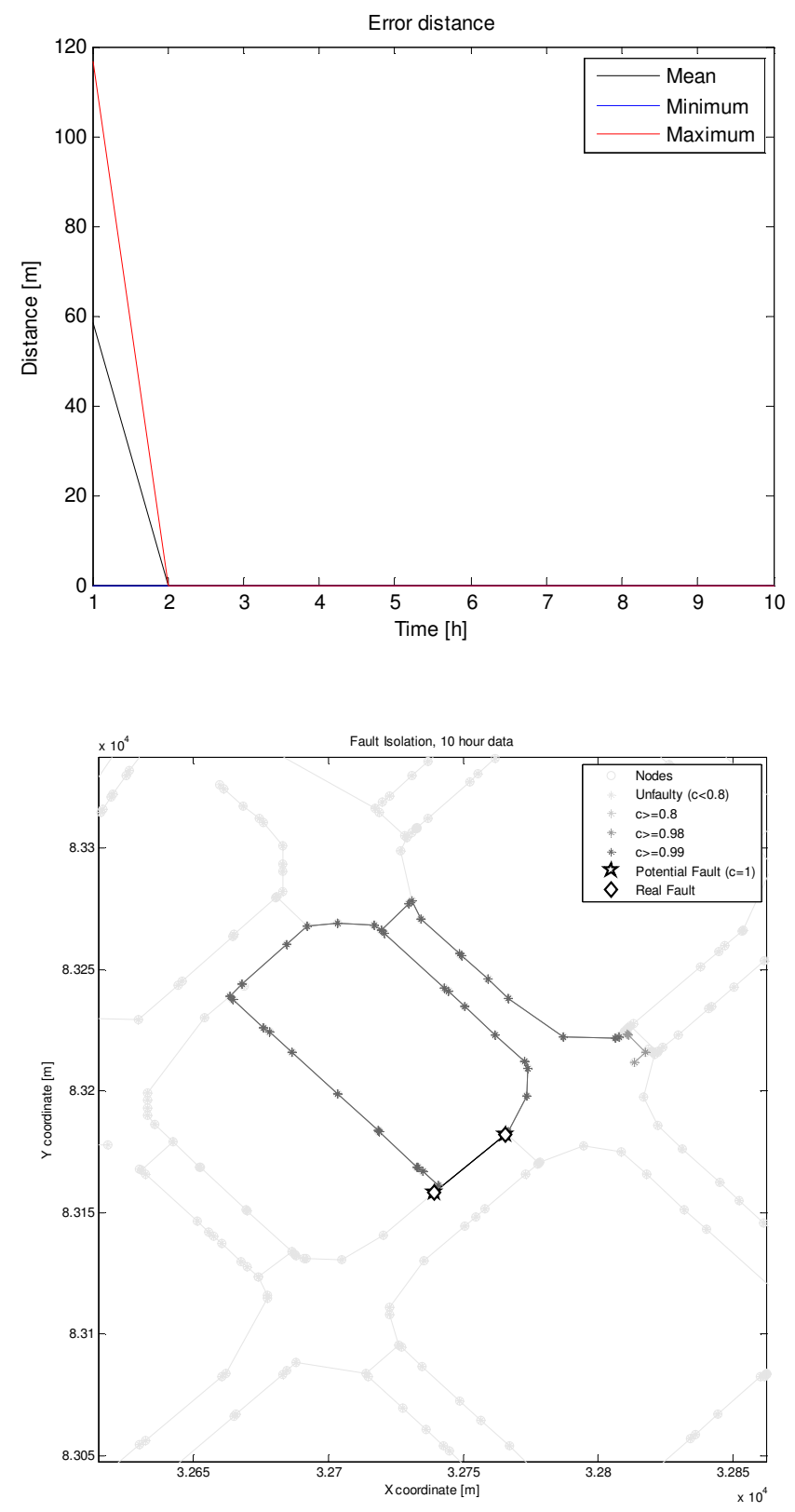

Figure 7. Error distance and geographic detail of chlorine bulk decay fault detection for the link TR00029794D at hour 48.

fault are depicted in Figs. 5-7. In the latters, the distance between the potential faulty links (starred in Figs. 5-7) and the actual faulty link (diamonds in Figs. 5-7) is represented. If more than one potential faulty link is obtained at a particular time, minimum, maximum and mean distances among potential faulty links set and actual faulty link are represented. For all cases, a good match between the actual and the identified fault has been achieved.

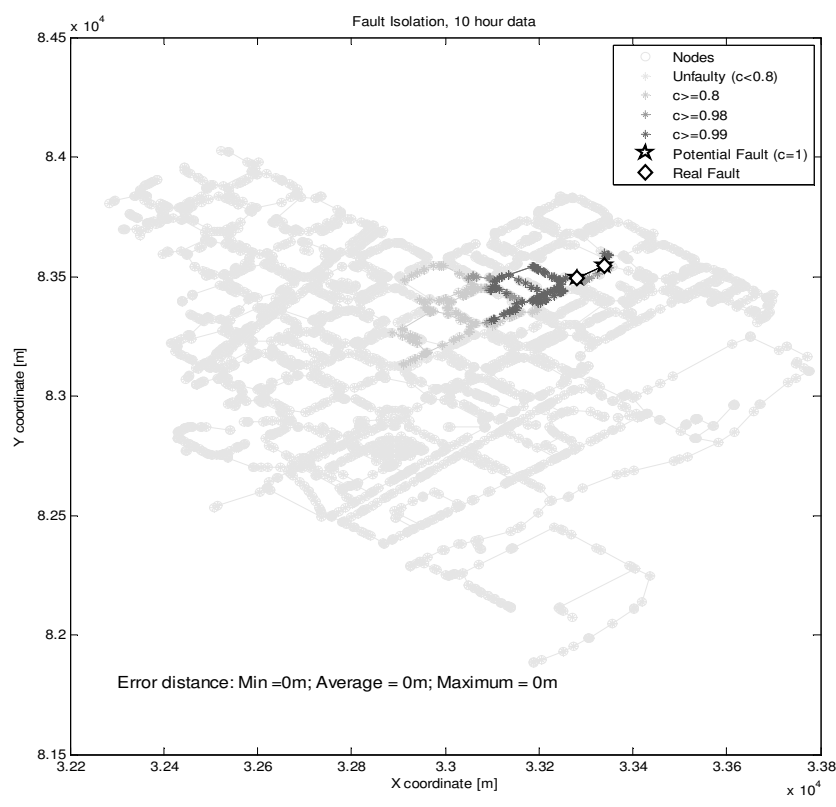

Figure 8. Chlorine bulk decay fault detection in link TR00353608D at hour 48 .

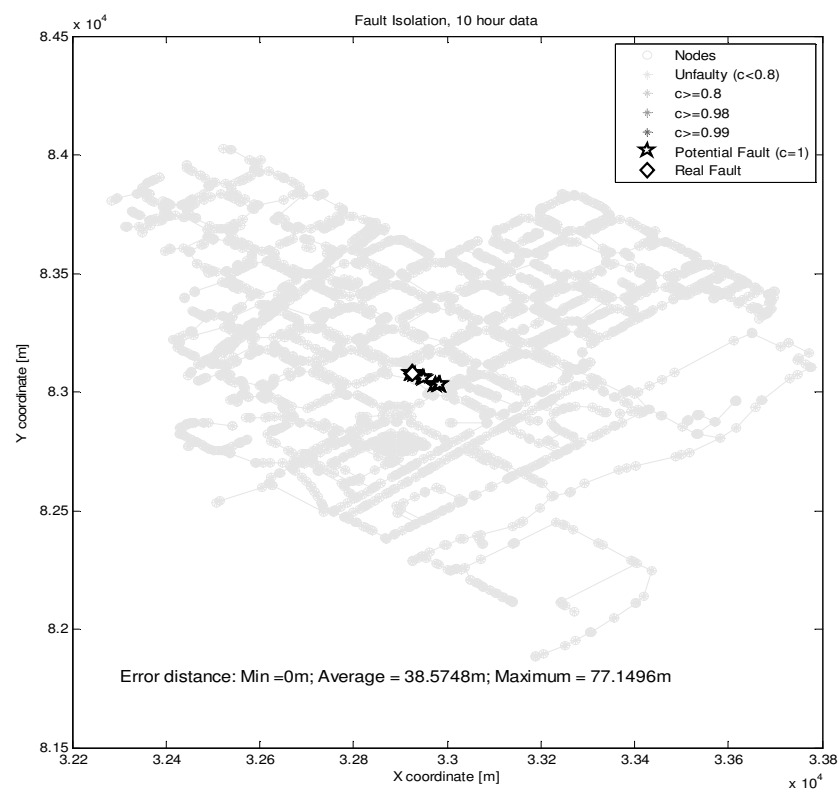

Figure 9. Chlorine bulk decay fault detection in link TR00029992A at hour 48 .

\section{Conclusions}

In this work, the problem of detection and localisation of water quality anomalies has been addressed. The abnormal water quality localisation method is based on the chlorine measurements and chlorine sensitivity analysis of nodes in a water distribution network. A fault localisation algorithm which correlates on line the residuals (generated by comparing the 


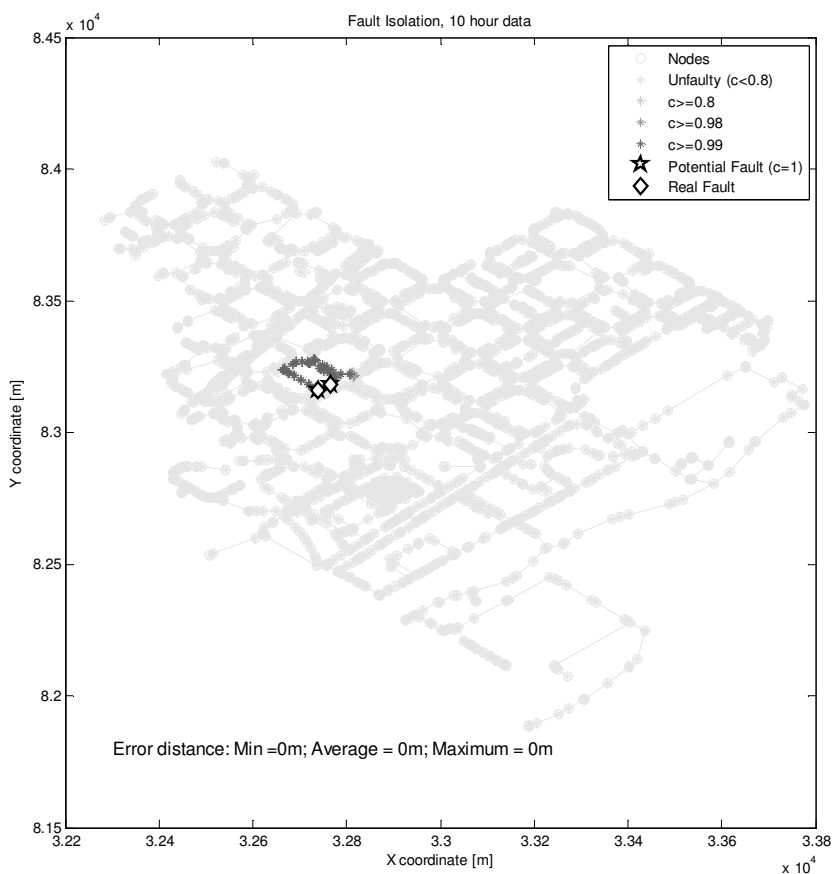

Figure 10. Chlorine bulk decay fault detection in link TR00029794D at hour 48.

available chlorine measurement with their estimation using a model) with the fault sensitivity matrix is used. The proposed algorithm has been applied in simulation to a DMA (Nova Icària) in the Barcelona network using EPANET software. The effectiveness of the method ensures the ability for a rapid-response to an abnormal event and, consequently, minimizes exposure risks to water consumers. The solution should help water companies to estimate the presence and the location of a bad chlorine concentration through a simple field data analysis.
Acknowledgements. This work was supported in part by the project RTNM AM0901 of Alliance, by the European Commission through contract i-Sense (ref. FP7-ICT-2009-6-270428) and the projects DPI2011-26243 (SHERECS) and DPI2009-13744 (WATMAN) of the Spanish Ministry of Economy and Competitiveness (MINECO).

Edited by: G. Fu

\section{References}

Blanke, M., Kinnaert, M., Lunze, J., and Staroswiecki, M.: Diagnosis and Fault-tolerant Control, 2nd Edn., Springer, 2006.

Gertler, J. J.: Fault Detection and Diagnosis in Engineering Systems, Marcel Dekker, 1998.

LeChevallier, M. W., Babcock, T. M., and Lee, R. G.: Examination and characterization of distribution system biofilms, Appl. Environ. Microbiol., 53, 2714-2724, 1987.

Lindley, T. R.: A framework to protect water distribution systems against potential intrusions, MS thesis, University of Cincinnati, Cincinnati, 2001.

Sadiq, R., Kleiner, Y., and Rajani, B.: Forensics of water quality failure in distribution system - a conceptual framework, Journal of Indian Water Works Association, 35, 267-278, 2003.

Pérez, R., Quevedo, J., Puig, V., Nejjar, F., Cugueró, M. A., Sanz, G., and Mirats, J. M.: Leakage Isolation in Water Distribution Networks: a Comparative Study of Two Methodologies on a Real Case Study, The 19th Mediterranean Conference on Control and Automation, 138-143, Corfu, Greece, 20-23 June 2011.

Quevedo, J., Puig, V., Cembrano, G., Blanch, J., Aguilar, J., Saporta, D., Benito, G., Hedo, M., and Molina, A.: Validation and reconstruction of flow meter data in the Barcelona water distribution network, Control Eng. Pract., 18, 640-651, 2010.

Quevedo, J., Cuguero, M. A., Pérez, R., Nejjari, F., Puig, V., and Mirats, J. M.: Leakage Location in Water Distribution Networks based on Correlation Measurement of Pressure Sensors, 8th IWA Symposium on Systems Analysis and Integrated Assessment, Watermatex 2011, San Sebastián, Spain, 20-22 June 2011. 